\title{
Iterative process control and sensor evaluation for deep drawing tools with integrated piezoelectric actuators
}

\author{
Tobias Bäume ${ }^{1, *}$, Wolfgang Zorn ${ }^{3}$, Welf-Guntram Drossel ${ }^{3}$, and Gerd Rupp ${ }^{2}$ \\ 1 Technology Development Tool Shop and Press Shop, Volkswagen AG, 38440 Wolfsburg, Germany \\ 2 Head of Tool Shop of the Volkswagen Brand, Volkswagen AG, 38440 Wolfsburg, Germany \\ 3 Fraunhofer Institute for Machine Tools and Forming Technology (IWU), 09126 Chemnitz, Germany
}

Received 30 October 2015 / Accepted 2 January 2016

\begin{abstract}
Due to the design-driven increase in complexity of forming car body parts, it becomes more difficult to ensure a stable forming process. Piezoelectric actuators can influence the material flow of stamping parts effectively. In this article the implementation of piezoelectric actuators in a large scale sheet metal forming tool of a car manufacturer is described. Additionally, it is shown that part quality can be assessed with the help of triangulation laser sensors, which are mounted on the blankholder. The resulting flange draw-in signals were processed and used to adopt the applying actuator force iteratively to reduce the occurrence of cracks. It was shown that process control helped to improve the quality of the stamping parts significantly.
\end{abstract}

Key words: Closed-loop control, Deep drawing, Process control, Piezo actuators, Production engineering, Sensor technology

\section{Introduction}

In sheet metal forming several parameters influence the quality of a part. If stable production conditions cannot be assured an open- or closed-loop feedback control can help to reduce rejected parts. Several feedback control approaches have been investigated to reduce the quantity of rejected parts which are caused by the adjusted process parameters.

As [1] showed it is possible to control a metal forming process if the material flow is monitored and a PID-controller varies the blank holder force. In this case a segmented blankholder is used to improve the quality of a square cup under laboratory conditions. Reference curves have been generated from sensor signals where the part quality was sufficient. Furthermore [2] designed another feedback control where master curves have been generated and unidirectional material flow sensors were recorded to monitor the process conditions. Adjustable nitrogen cylinders were controlled to regulate the blank holder force distribution of a modified wheel house liner tool. In reference [3] also used the material flow as sensor signal to control the blankholder force. Here a fuzzy control has been developed which simplifies the modelling due to a less accurate model which is adequate enough. In reference [4] examined another specially adopted tool where the active area of the blank holder

*Corresponding author: tobias.baeume@volkswagen.de is mounted on piezoelectric sensors to measure the dynamic force which is due to the sliding sheet metal between die and blankholder. The measured force signal can be converted into dynamic friction coefficients. Four embedded cavities, which vary blank holder force regions locally, are used by Endelt and Dankert [5] to control a sheet metal forming process. A cascade control was designed. The inner loop is used to minimize a linear-quadratic regulator (LQR) cost function (LQR optimal control). The outer loop reduces long term disturbances with the help of a linear learning algorithm. Due to the modified tool the concept is hardly implementable into serial production tools. For all of these concepts, a specially designed tool is needed in order to enable a local alternation of the blank holder force distribution.

This work will show an additional approach where piezoelectric actuators. The approach was derived from the reference [14]. Here, multiple arranged piezoelectric actuators where used to influence the material flow. An experimental tool was used to verify the effectiveness of this concept. A cover plate protects the actuators and will transfer the applied force. The followed working principle can be seen in Figure 1. This concept was taken and the actuators where embedded into the die of a large scale sheet metal forming tool. Additionally the actuators were controlled to vary the blankholder force distribution, which have not been investigated before. Different 

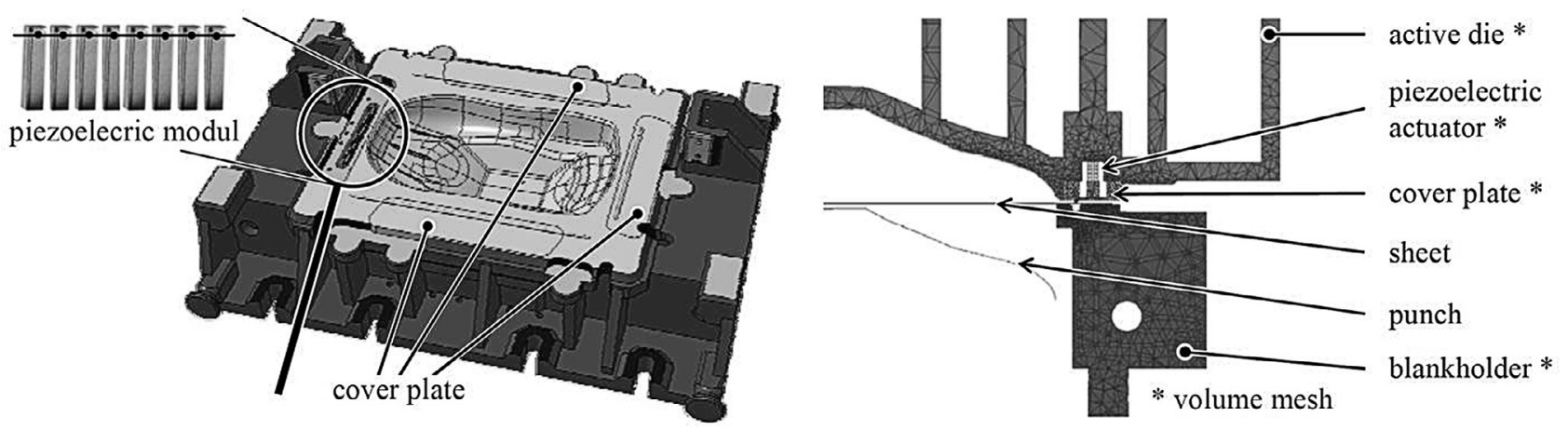

Figure 1. Working principle and set-up of piezoelectric actuators in metal forming tools [14].

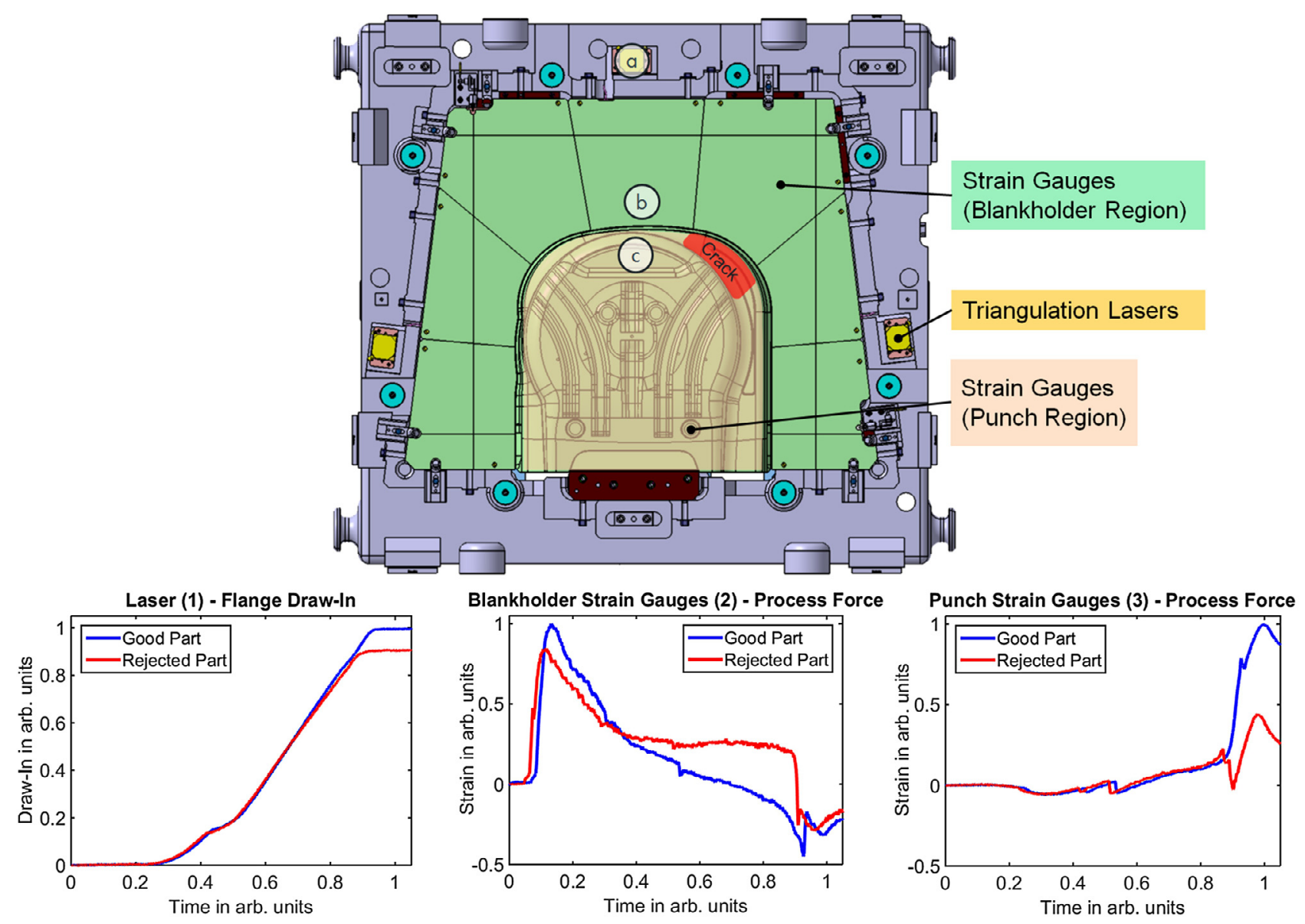

Figure 2. Preliminary analysis of a triangulation laser (a), blankholder strain gauge (b) and punch strain gauge (c) sensor to evaluate the technical suitability as control.

sensors were analysed in advance to obtain an optimized control variable for the presented closed loop control approach.

\section{Set-up}

To design a process control sensors are necessary to distinguish the parameters which correlate with part quality. Several sensors for different mode of failures have been investigated in sheet metal forming. The lower boundary of the forming process can be analysed with sensors tested by Griesbach [1],
Blaich and Liewald [6] and Straube [7]. If the sensor signals alter from the reference signals then wrinkling will arise and the blankholder force has to be increased. The upper process limit is where necking, tearing or cracking occurs. Kergen and Jodogne [8] shows that strain gauges are feasible to detect those mentioned failures. Beside inductive displacement sensors [9] for detecting the change of material properties the contact-less measurement of the flange draw-in via triangulation lasers are a suitable alternative for process quality evaluation [10]. 


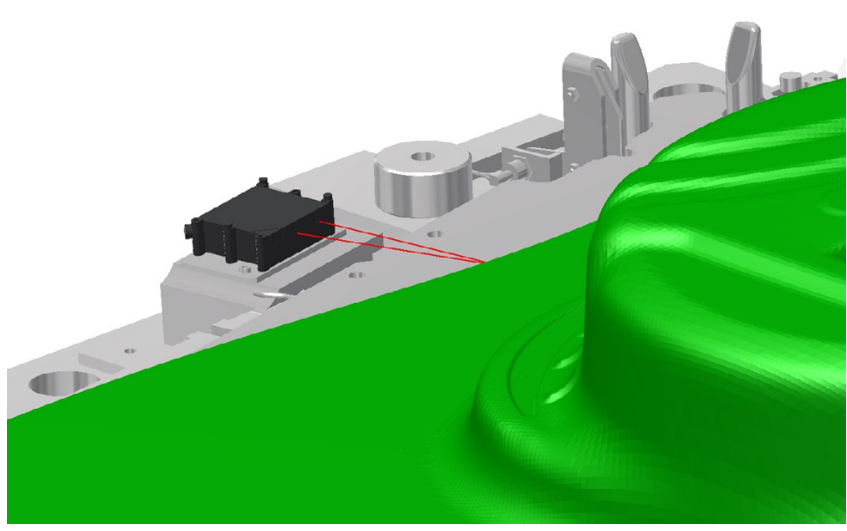

Figure 3. Mounted triangulation laser L2 and working principle.

These aforesaid measurement methods have been analysed with regards to their ability to be implemented into the iterative process for control strategies. Therefore different types of sensors have been integrated into a forming tool, as suggested by Waltl [12] (see Figure 2). For each sensor the corresponding exemplarily signals are illustrated in Figure 2. Sensor (b) and (c) where implemented as suggested by Kergen and Jodogne [8] and represent the strain of the affected tool areas caused by the acting blankholder/process force. Sensor (a) was used to measure the flange draw-in as [10] proposed. The signal path of a good and one rejected part were chosen exemplarily to amplify and clarify the difference in signal characteristics. The area of crack occurrence can be seen in Figures 2 and 11. Wrinkling has not been considered.

Due to the die cushion, which applies the blankholder force to the blankholder, Sensor (b) represents the compression/ strain in that region. Process variation will influence the signal qualitatively as Figure $2 b$ shows. Here, the acting force will remain in the localised area where the crack occurs. Thus the sensor is suitable to detect process variations. Additionally it is possible to sense exceed process limits only if the sensor is mounted close to the area of failure.

During the forming process the blank will be stretched over the punch. Moderate process changes will not affect the signal characteristics reliably. If a crack occurs the acting force cannot be transferred anymore, which causes an abrupt signal drop. Hence, mostly the signal paths in Figure 1c are congruent until a crack occurs at approximately $85 \%$ of the process time. Consequently this sensor represents a binary signal characteristic. Closer proximity to the crack initialization increases the signal sensitivity. Therefore the sensor is useful to recognize material failure locally.

The flange draw-in represents the material flow during the stroke. As Figure 2a illustrates, sensor signals weakly start to diverge when small process fluctuations appear. Furthermore, the signal starts to differ when the load/strain limits of the material are exceeded and tearing begins. The material flow will differ significantly when a crack occurs which reduces the total draw-in (see Figure 2a at approximately $85 \%$ of the process time). The large process variation can cause an early diverge of the signals. In both cases the total flange draw-in will differ from signals of normal process behaviour. As a result, the flange draw-in was identified as parameter which strongly correlates with part quality [11]. In contrast to wrinkling and crack detecting sensors flange draw-in sensors allow to estimate the part quality without exceeding the process limits. Regarding the requirements to fulfil the large scale production conditions, the triangulation laser was used for further investigation.

Due to the small dimensions the laser can be flexibly mounted on the outer regions of the blank holder as Figure 3 illustrates. That allows replacing the sensor if the error pattern, which was predicted from sheet metal forming simulation, differs from reality. Finally the triangulation laser provides reliable measure signals with an accuracy of less than $0.1 \mathrm{~mm}$.

The forming tool was supplemented by five piezo actuator modules, which are used to redistribute the pressure in the flange area of the blankholder, as suggested by Mainda [13]. The actuator modules corresponding power amplifiers are arranged within the tool. The energy supply and the control signals are induced via an additional interface mounted on the tool. This interface is also used for the sensor signals. The control processing was firstly realized on a rapid control prototyping system, which was arranged outside of the forming machine. This allows a convenient reconfiguration of the controller without stopping the process.

\section{Control}

\subsection{Basics}

An arbitrary process $\boldsymbol{\Psi}$, which connects an input vector $\mathbf{x}$ with an output vector $\mathbf{z}$ is defined as

$$
\mathbf{z}=\Psi(\mathbf{x})
$$

where $\mathbf{x}$ and $\mathbf{z}$ contain several single variables with the dimensions $n$ and $m$ respectively:

$$
\begin{aligned}
& \mathbf{x}=\left(x_{1}, x_{2}, \ldots x_{n}\right) \\
& \mathbf{z}=\left(z_{1}, z_{2}, \ldots z_{m}\right)
\end{aligned}
$$

For each output variable $z_{i}$ a functional relation can be defined. The type of initial function mainly depends on the non-linearity of the process. If there is no information of the process, a polynomial approach should be a feasible possibility of reflecting the process. Within the scope of the presented examinations a second-order polynomial was applied:

$$
\begin{aligned}
z_{i}= & a_{i, 0}+a_{i, 10} \times x_{1}+a_{i, 20} \times x_{2}+\ldots+a_{i, 12} \times x_{1} \times x_{2} \\
& +a_{i, 11} \times x_{1}^{2}+a_{i, 22} \times x_{2}^{2}+\ldots
\end{aligned}
$$

The coefficients $a_{i, j}$ are calculated with a regression analysis of the results, derived from simulations or metrological examinations (see section 0). Using Eqs. (1) and (4) an analytical description of the deep drawing process with specified input and output parameters could be obtained.

In Figure 4 a schematic representation of the target area of error-free parts depending on the output parameters $\mathbf{z}_{i}$ is given. 


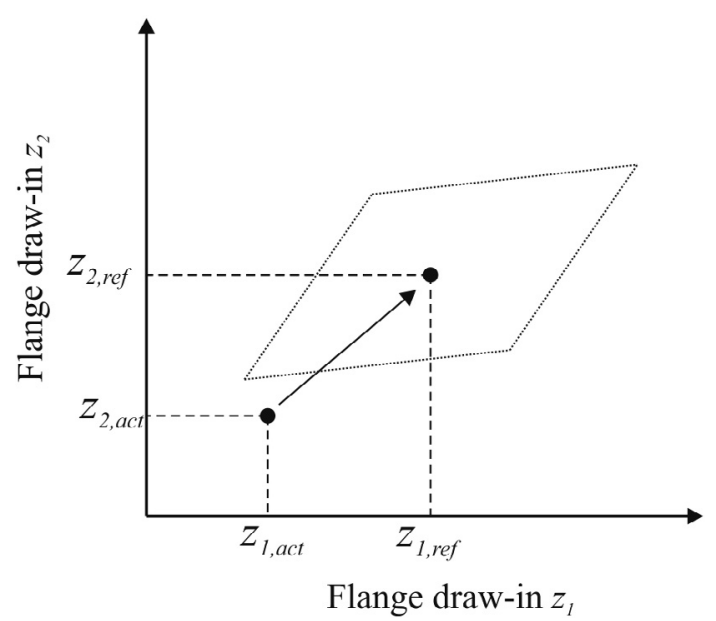

Figure 4. Target area of error-free parts.

$\mathbf{z}_{\text {act }}$ and $\mathbf{z}_{\text {ref }}$ represent the actual output values of an arbitrary production step and the given reference values respectively.

To help ensure a stable process the output values should coincidence with the reference values. Due to systematic and statistic errors there is a difference between the actual and the reference values. The reasons for this are not covered by the regression model in any case. To minimize the gap a Taylor expansion in the topical operating point $\bar{x}$ can be done for $\mathbf{z}$.

$$
\begin{gathered}
\Delta z_{1}=\left[\left.\frac{\partial z_{1}}{\partial x_{1}}\right|_{x=\overline{\boldsymbol{x}}} \cdot \Delta x_{1}\right]+\left[\left.\frac{\partial z_{1}}{\partial x_{2}}\right|_{x=\overline{\boldsymbol{x}}} \cdot \Delta x_{2}\right]+\ldots\left[\left.\frac{\partial z_{1}}{\partial x_{n}}\right|_{x=\overline{\boldsymbol{x}}} \cdot \Delta x_{n}\right] \\
\Delta z_{2}=\left[\left.\frac{\partial z_{2}}{\partial x_{1}}\right|_{x=\overline{\boldsymbol{x}}} \cdot \Delta x_{1}\right]+\left[\left.\frac{\partial z_{2}}{\partial x_{2}}\right|_{x=\overline{\boldsymbol{x}}} \cdot \Delta x_{2}\right]+\ldots\left[\left.\frac{\partial z_{2}}{\partial x_{n}}\right|_{\boldsymbol{x}=\overline{\boldsymbol{x}}} \cdot \Delta x_{n}\right] \\
\vdots \\
\Delta z_{m}=\left[\left.\frac{\partial z_{m}}{\partial x_{1}}\right|_{\boldsymbol{x}=\overline{\boldsymbol{x}}} \cdot \Delta x_{1}\right]+\left[\left.\frac{\partial z_{m}}{\partial x_{2}}\right|_{\boldsymbol{x}=\overline{\boldsymbol{x}}} \cdot \Delta x_{2}\right]+\ldots\left[\left.\frac{\partial z_{m}}{\partial x_{n}}\right|_{x=\overline{\boldsymbol{x}}} \cdot \Delta x_{n}\right]
\end{gathered}
$$

This linear system of equations can be expressed by

$$
\Delta \mathbf{z}=\mathbf{J}_{\mathbf{A}} \cdot \Delta \mathbf{x}
$$

The difference $\Delta \mathbf{z}$ between actual and reference values is known from the process, the matrix $\mathbf{J}_{\mathbf{A}}$ with its coefficients can be derived from the process model. By transforming of equation (6) the necessary modification $\Delta \mathbf{x}$ of the operating point can be calculated:

$$
\Delta \mathbf{x}=\mathbf{J}_{\mathbf{A}}^{-1} \cdot \Delta \mathbf{z}
$$

In general the number of input parameters does not correlate with the number of output parameters. Thus, $\mathbf{J}_{\mathbf{A}}$ is a nonsquare matrix in most cases, which requires a pseudoinverse to calculate $\Delta \mathbf{x}$. This allows programming a controller, which uses the current operating point and the analytical model. The corresponding scheme of the closed loop control is shown in Figure 5.

To illustrate this approach two scenarios were applied. In the first scenario the convergence behaviour was examined by assuming a random-parametrized model with two dimensions. Due to the non-linear process model the calculated $\Delta \mathbf{z}$

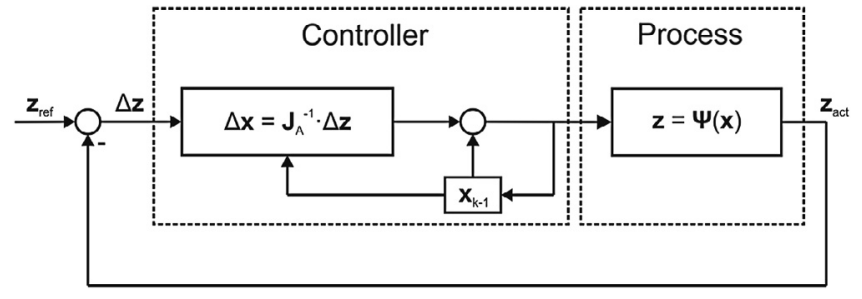

Figure 5. Control loop.

does not necessarily match the output $\Delta \mathbf{z}$. This requires an additional damping factor and several steps to approach the reference point. The second scenario provides a disturbed process. The controller tries to keep the output variable at the stable point, while an increasing offset manipulates the process. Figures $6 \mathrm{a}$ and $6 \mathrm{~b}$ show corresponding simulation results for both scenarios.

\subsection{Design of experiments and process model}

As already stated the process model of the deep-drawingprocess is represented by the analytic equations for the flange draw-in depending on the control parameters of the piezo actuators. Each equation satisfies the structure given in equation (4). To determine the parameters $a_{i, j}$ a regression analysis was used with the usage of a statistical design of experiments. As variable parameters the amplitude of the actuation voltage of each piezo actuator was chosen. To reduce the range of the design a time variant voltage was not taken into consideration. The design of experiments (DOE) was achieved with computational assistance using the commercial software Cornerstone. For each of the five input parameters three categories (low, mean, high) were given. As a result of the DOE a total number of 26 input vectors were suggested as grid points for deriving the process model using the multi-dimensional regression analysis. For clarification, Figure 7 reduces the mentioned principle to a one-dimensional plot with reference to the consideration of the $x$-values as $n$-dimensional values. The regression curves for all three flange draw-ins provide the coefficients $a_{i, j}$ (see Eq. (4)), which were obtained using the beforementioned software Cornerstone.

It was established that conventional industrial deep drawing simulations have some limitations. The models with rigid tool surfaces and Coulomb friction models cannot reproduce the interaction between actuators and the reaction of the forced-controlled drawing cushion/cushion bead. Therefore investigated FEM-based models differ significantly from the analysed empirical ones. Further investigations with refined simulation models could improve the quality of the process model. A volumetric mesh of the full tool is advised to consider the operating principle of the actuators with respect to the effects on the stiffness of the blankholder [11, 13]). Considering this the empirical models have been used for further analysis.

For the first experimental set-up a forming press with multi-point die/drawing cushion has been implemented. Here sixteen hydraulic cushion pins were distributed evenly on the 
(a)

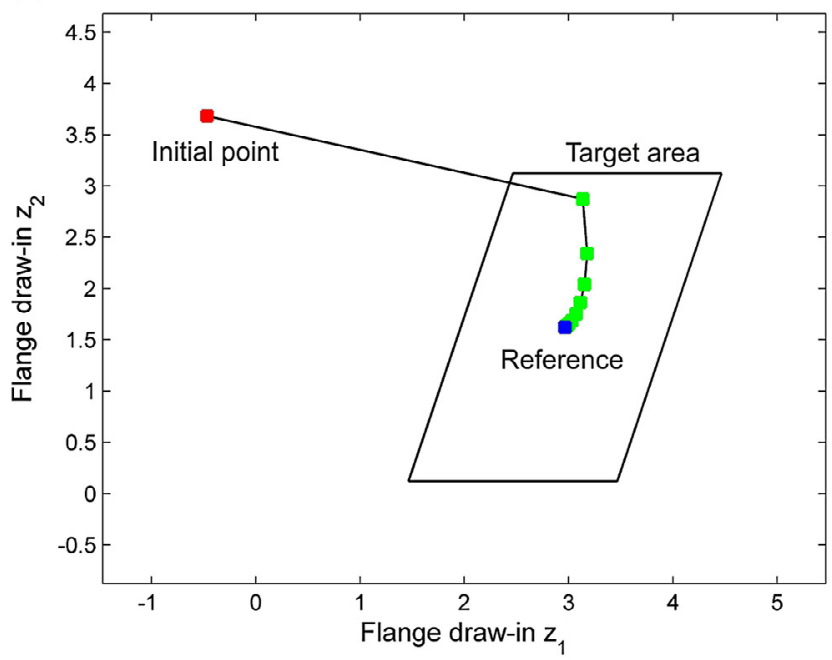

(b)

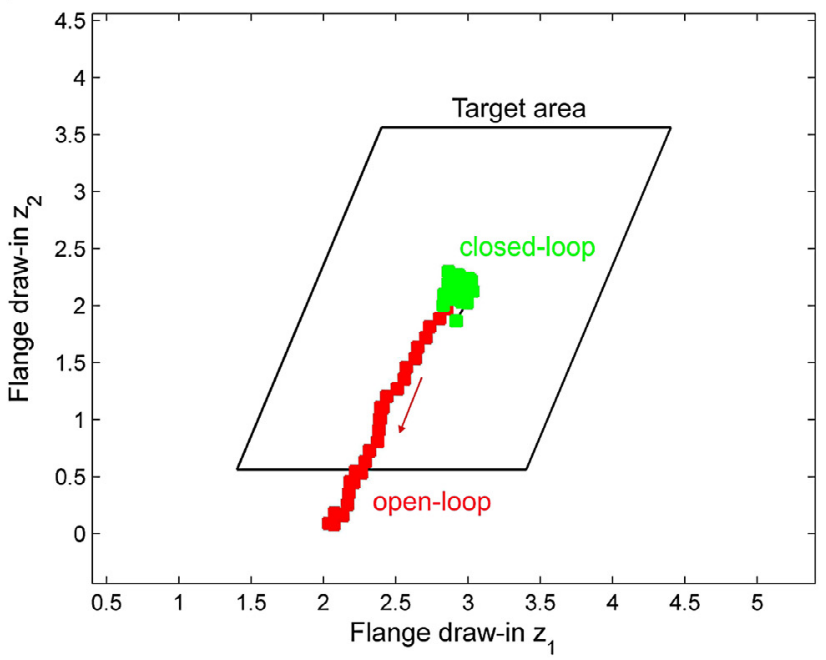

Figure 6. Simulation results for two convergence behaviour scenarios (a) and (b).

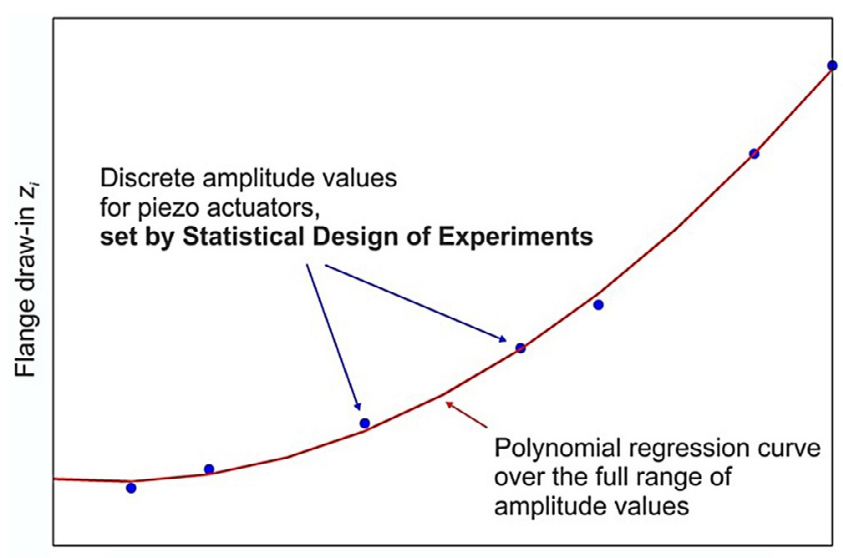

Input vector $\mathbf{x}$ (Vector of piezo actuator amplitude values)

Figure 7. Principle of a regression analysis based on grid points derived from a statistical design of experiments.

inner area of the blankholder to apply the force homogeneously. The analysis of the calculated parameters $a_{i, j}$ have shown several effects of cross correlation. Thus, a single actuator does not just affect the peripheral area, but also a flange draw-in in other sectors of the blank (see Table 1). One reason for such occurrence could be the inner elastic and plastic bonding of the forming material. This will cause changes in actuator forces and in certain active areas it will affect the material flow of the surrounded area as well.

To clarify the cross correlation, the process model has been visualized in Figure 8. Actuator P1 and P3 are plotted to illustrate the aforementioned effects. All other actuators are turned off. The initial draw-in plane shows the zero level of the flange draw-in from laser 2 when all actuators are deactivated.

As Figure 8 indicates, actuator P1 will affect the material flow of the distant area from laser 2. Paraphrased, P3 inhibits the material flow which causes a reduced draw-in. P1 induces
Table 1. Extract of the coefficient table.

\begin{tabular}{lccc}
\hline Term & Laser 1 & Laser 2 & Laser 3 \\
\hline $\mathrm{U}_{\max , \mathrm{P} 1}$ & - & 0.005858 & -0.02109 \\
$\mathrm{U}_{\max , \mathrm{P} 3}$ & 0.00627 & -0.00486 & 0.00477 \\
$\mathrm{U}_{\max , \mathrm{P} 1} * \mathrm{U}_{\text {max }, \mathrm{P} 3}$ & -0.00012633 & -0.00014544 & -0.00012679 \\
\hline
\end{tabular}

a contrary effect. Furthermore, due to reciprocal effects, this retarding impact will be reinforced if both actuators work simultaneously. This will allow combining several actuators to maximize the efficiency.

A try-out press with eight-point drawing cushion was used to obtain a second empirical process model. The determined parameters $a_{i, j}$ are comparable in their qualitative appearance. Divergences of the interacting actuators can be explained by the elasticity of the forming tool and the use of a die cushion, where one pressure cylinder acts on more than one barrels of the tool. This leads to a decrease in the contact pressure of the implied peripheral areas. Therefore the affected areas depend on the selected die cushion. In addition, quantitative results of the model-based flange draw-in are not able to be compared due to different total blankholder forces and the mentioned deviating amount of cross correlation. Furthermore, the obtained draw-in values of both experimental set-ups cannot be compared because of a gradient-based controller. Relative deviations of the flange draw-in are used to calculate the relative response of the actuator force. Due to similar model characteristics no additional analysis is presented.

For evaluating the sensor signal quality regarding the suitability for a closed-loop control the parts were individually examined. Here, only the parts which were drawn on the press with multi-point die cushion where taken into account. For several quality conditions (without errors, necking, cracks) the range of occurred draw-in-values was considered. Figure 9 shows the corresponding box-plots for each laser sensor. There were overlaps of all considered quality conditions. The overlap 


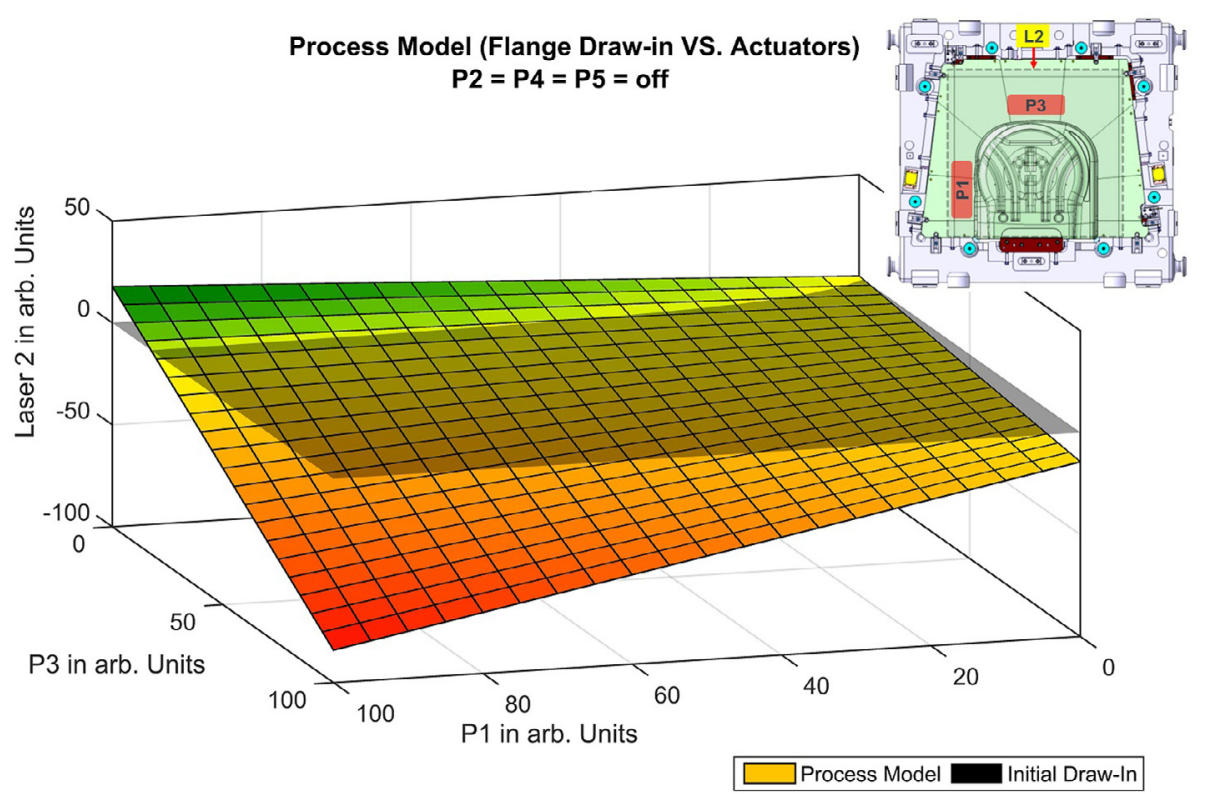

Figure 8. Visualized process model for actuator P1 and P3 over L2.

(a)

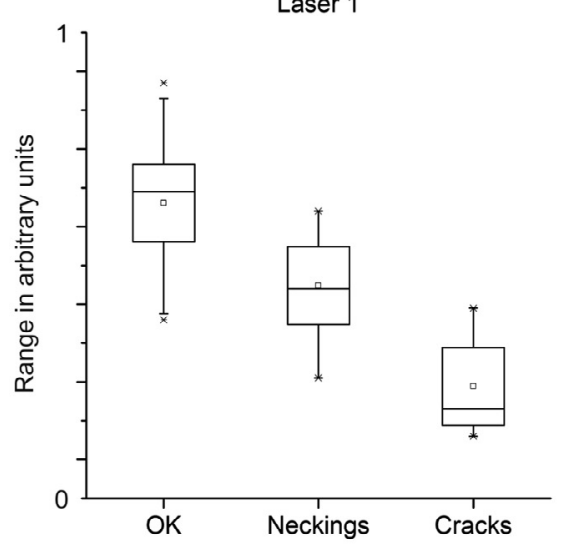

(b)

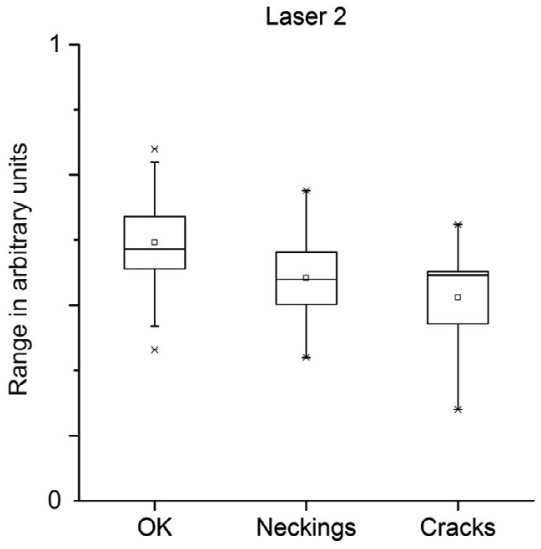

(c)

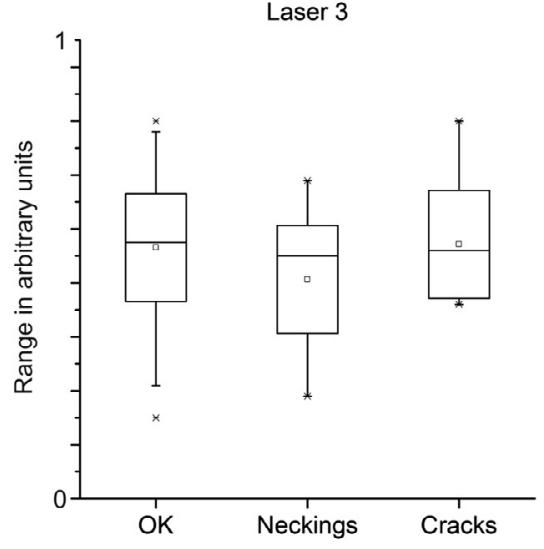

Figure 9. Box-plot of the measured values.

(a)

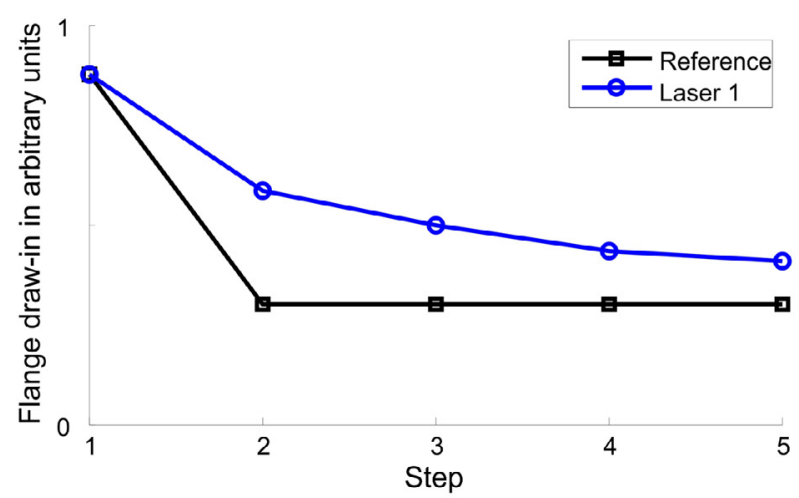

(b)

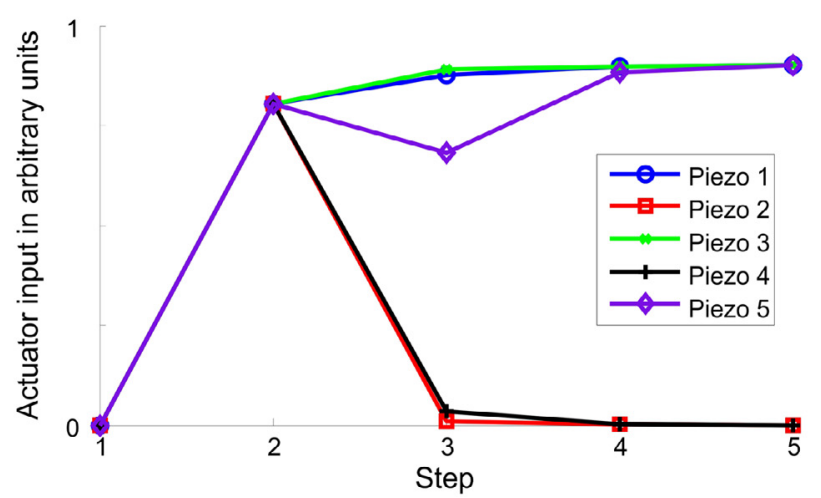

Figure 10. Draw-in values (a) and actuator input (b) of a step-by-step control. 


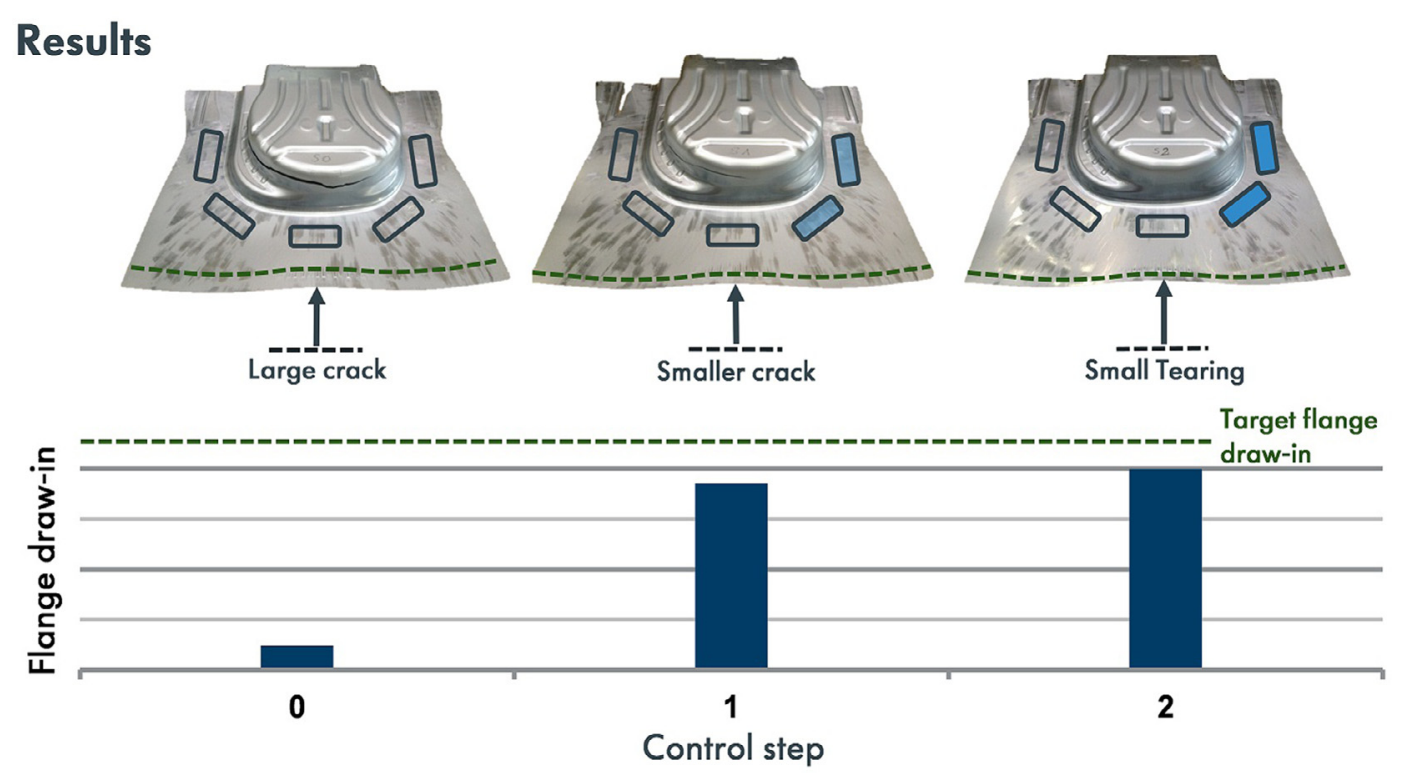

Figure 11. Results of the step-by-step control.

for laser 2 and laser 3 is rather huge significant, whereas the overlap for laser 1 allows a quite good allocation of draw-in values to the part quality. Hence, only the signal of laser 1 is suitable for a closed-loop control.

\subsection{Results}

To validate the functionality of this control approach several scenarios were investigated. Due to the aforementioned reasons these scenarios only consider laser 1 as input parameter. Figure 10 shows the results for a step-by-step control of the draw-in of the blank. After an initial step the reference value was decreased. The controller calculated the new actuation forces of the five piezo actuators. Clearly visible is the stepwise convergence of the measured and actuating values. This series was intended to investigate the principle control behaviour of this approach. Assuming a larger range of operation the difference between actual value and reference value should vanish.

In another scenario the tool was adjusted to produce a large crack in the part front with no driven piezo actuators. To avoid the crack the reference value of laser 1 was increased to result in a more intensive flow of the material. As shown in Figure 11 the controller takes three steps to minimize and eliminate the crack.

\section{Summary}

Due to the design-driven increase in complexity of forming car body parts, it becomes more difficult to ensure a stable forming process. Piezoelectric actuators can influence the material flow of stamping parts effectively by redistribute the forces in the tool. In this article the implementation of piezoelectric actuators in a large scale sheet metal forming tool of a car manufacturer was described. Additionally, it was shown that part quality can be assessed with the help of triangulation laser sensors, which are mounted on the blankholder. The resulting flange draw-in signals were used to reduce the occurrence of cracking. An iterative process control was developed to minimize the current flange draw-in compared to a reference signal. The applied piezoelectric actuator force will be adopted after each stroke to influence the blankholder force distribution. The blankholder force of the die cushion was not modified. If the reference flange draw-in will be set to the upper process boundaries wrinkling was minimized automatically. A resulting analytical process models from a multi-point and eight-point die cushion press were derived. The origins are accountable to the regression analysis based on an experimental design. The obtained process models were comparable in their qualitative characteristic. Deviations occur due to different operating behaviour and the resulting/caused changed cross correlation. Because of this further investigation regarding to the interaction between actuators and die cushion is recommended. Finally, it was shown that process control helped to improve the quality of the stamping parts significantly.

\section{Disclaimer}

The results, opinions or conclusions of this publication are not necessarily those of the Volkswagen AG.

\section{References}

1. B. Griesbach, Fortschritt-Berichte VDI 547 (2000).

2. S. Rittmeier, Systemunterstützte Umformung, $\mathrm{PhD}$ thesis, Technische Universität Dresden, Cuvillier, Göttingen, 2007.

3. J.-W. Yun, Berichte aus dem IFUM 2 (2005). 
4. M. Ziegler, Prozessregelung zur Regelung der Reibungskraft, in: K. Siegert (Ed.), Neuere Entwicklungen in der Blechumformung, 1998, pp. 427-446.

5. B. Endelt, J. Danckert, International Journal of Material Forming 3 (2010) 25-28.

6. M. Liewald, S. Wagner, J. Hengelhaupt, M. Vulcan, New Approaches for the Closed Loop Control of the Deep Drawing Process of Extensive Sheet Metal Parts. 7, in Internationales Stuttgarter Symposium 2007, Automobil- und Motorentechnik - FKFS, Stuttgart, Germany, 2007, pp. 611-626.

7. O. Straube, Produktionstechnik 152 (1994).

8. R. Kergen, P. Jodogne, Computerized control of the blankholder pressure on deep drawing presses, SAE-papers 920433, 1992.
9. U. Forstmann, Induktive Wegsensoren zur Überwachung und Regelung des Blecheinzugs beim Tiefziehen, E. Uhlmann (Ed.), Berichte aus dem Produktionstechnischem Zentrum Berlin, Fraunhofer IPK, Berlin, 2000.

10. H. Bräunlich, Berichte aus dem IWU 19 (2002).

11. V. Thoms, M. Schatz, EFB-Forschungsbericht 278 (2008).

12. H. Waltl, 3rd International Conference on Accuracy in Forming Technology, pp. 9-16, 2009.

13. P. Mainda, Berichte aus dem IWU 68 (2012).

14. R. Neugebauer, Integrated piezoelectric actuators in deep drawing tools, Proc. SPIE 7979, 2011.

Cite this article as: Bäume T, Zorn W, Drossel W-G \& Rupp G: Iterative process control and sensor evaluation for deep drawing tools with integrated piezoelectric actuators. Manufacturing Rev. 2016, 3, 3. 